Review article

\title{
EGFR inhibition in NSCLC: New findings. ... and opened questions?
}

\section{Francesco Passiglia ${ }^{1}$, Angela Listì ${ }^{1}$, Marta Castiglia, Alessandro Perez, Sergio Rizzo, Viviana Bazan, Antonio Russo*}

Department of Surgical, Oncological and Oral Sciences, Section of Medical Oncology, University of Palermo, Palermo, Italy

\section{Contents}

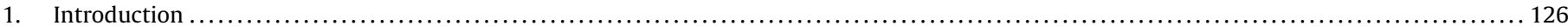

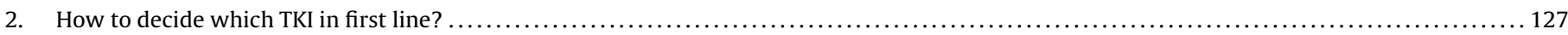

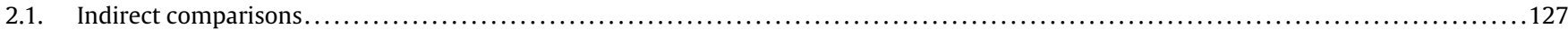

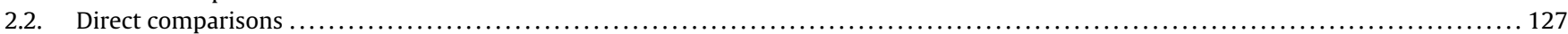

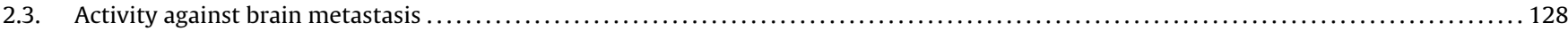

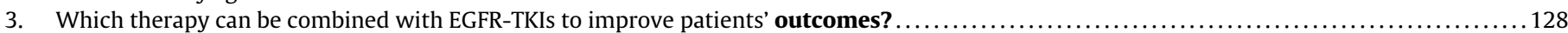

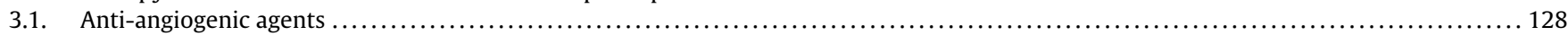

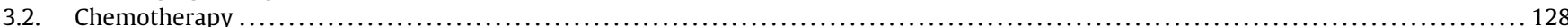

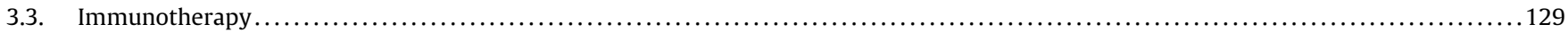

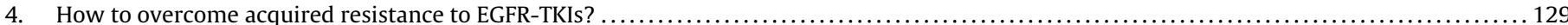

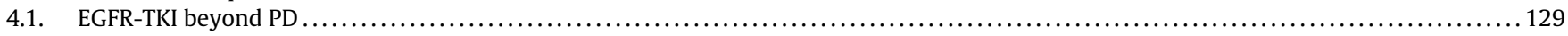

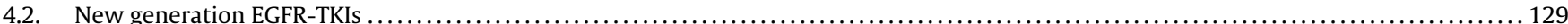

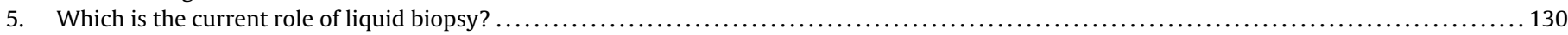

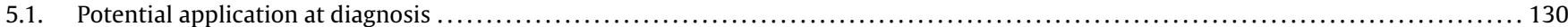

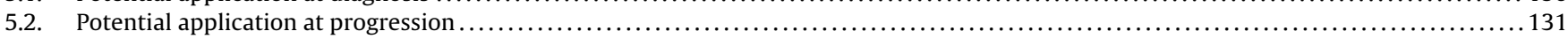

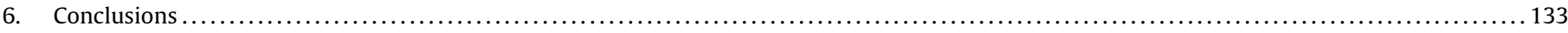

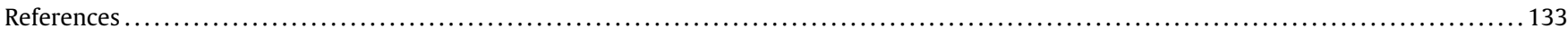

\section{A R T I C L E I N F O}

\section{Article history:}

Received 16 January 2017

Received in revised form 14 February 2017

Accepted 14 February 2017

\section{Keywords:}

EGFR

Targeted therapy

TKIs

Resistance

Liquid biopsy

\begin{abstract}
A B S T R A C T
The targeted inhibition of epidermal growth factor receptor (EGFR) has represented a milestone in the treatment of lung cancer. Several studies convincingly and consistently demonstrated a significant superiority of EGFR-TKIs over standard platinum-chemotherapy in EGFR-mutated NSCLC patients, leading to the sequential approval of gefitinib, erlotinib and afatinib as new standard first-line clinical treatment. To date we are witnessing a second revolution in the management of EGFR-positive NSCLC thanks to the development of new treatment strategies aiming to overcome acquired resistance to TKIs and ultimately improve patients' outcomes. In this review we summarize the most important recent findings regarding EGFR-inhibition in NSCLC, highlighting the current unsolved questions on the selection of the best TKI in first-line, which therapy can be combined with upfront EGFR-TKIs, how to overcome acquired resistance, and which are the clinical applications of liquid biopsy.
\end{abstract}

(c) 2017 Elsevier B.V. All rights reserved.
* Corresponding author at: Section of Medical Oncology, Department of Surgical, Oncological and Oral Sciences, Palermo University Hospital, Via del Vespro 129, 90127, Palermo, Italy.

E-mail address: antonio.russo@usa.net (A. Russo).

1 These authors equally contributed to this work.

\section{Introduction}

The pharmacological inhibition of the epidermal growth factor receptor (EGFR) by tyrosine-kinase inhibitors (TKIs) represented the first example of successful targeted therapy in lung cancer. The discovery of EGFR activating mutations in 2004 as oncogene driver in a subgroup of patients with non-small cell lung cancer (NSCLC) led to the development of a new family of biological agents, called EGFR-TKIs, which were able to selectively bind and 
inhibit the EGFR molecular pathway (Bronte et al., 2014). Since the approval of the first EGFR-TKI gefitinib in clinical setting, several other compounds have been developed by pharma, which may be classified as first, second, and third-generation EGFR-TKIs. About eight phase III randomized clinical trials compared EGFR-TKI Gefitinib, Erlotinib, or Afatinib vs platinum-based chemotherapy as first-line treatment for EGFR-mutated NSCLC patients (Reis-Filho and Pusztai, 2011; Mitsudomi et al., 2010; Maemondo et al., 2010; Paz-Ares et al., 2017a; Network CGA, 2012; Zhou et al., 2011; Sequist et al., 2011; Wu et al., 2014). The results of all such studies convincingly and consistently demonstrated a significant superiority of EGFR-TKIs over standard platinum-chemotherapy, improving both response rate (RR), progression free survival (PFS) and quality of life (QoL) of EGFR-mutated NSCLC patients. These positive results have led to the sequential approval by regulatory authorities of gefitinib, erlotinib and afatinib as upfront treatment of EGFR-mutated NSCLC patients. The advent of targeted agents in clinical setting has certainly represented one of the most important innovations in the treatment of lung cancer over the last years. These drugs have revolutioned the clinical management of about 40\% Asian and 12\% Caucasian NSCLC patients harboring EGFRmutations, whose survival outcomes nearly doubled compared to standard chemotherapy. However, the development of resistance has significantly limited the potential benefit of these drugs. Indeed the majority of patients have a disease progression (PD) within one year of TKI therapy (Passiglia et al., 2015). Recent studies suggested new treatment strategies to overcome resistance and ultimately improve survival outcomes of EGFR-mutated patients, thus leaving some questions unsolved:

- How to decide which TKI in first line?

- Which therapy can be combined with EGFR-TKIs to improve patients outcomes?

- How to overcome acquired resistance to EGFR-TKIs?

- Which is the current role of liquid biopsy?

In this review we highlight all most recent important findings regarding EGFR-inhibition in NSCLC, trying to provide evidencebased answers to the aforementioned questions.

\section{How to decide which TKI in first line?}

\subsection{Indirect comparisons}

To date we have three EGFR-TKIs approved as first-line treatment for EGFR-mutated NSCLC patients. Two first-generation, reversible TKIs, gefitinib and erlotinib, and one second-generation, irreversible, pan-Her TKI, afatinib. These compounds have shown similar efficacy in studies and meta-analysis which indirectly compared them, reaching an efficacy plateau in both PFS (10-12 months) and OS (ranging from 21 to 30 months) (Haspinger et al., 2015). Afatinib is the only compound showing a significant os benefit in the recent pooled analysis of both LuxLung3 (LL3) and LuxLung6 (LL6) trials, even if limited to the subgroup of patients with EGFR exon 19 deletion (Yang et al., 2015a). Furthermore it is the TKI with more evidence-based activity against EGFR uncommon mutations (Yang et al., 2015c). Particularly it showed to be active against the most frequent uncommon mutations, including Gly719Xaa, Leu861Gln, and Ser768Ile, while very low activity has been demonstrated in other mutation types, such as de-novo Thr790Met mutation or insertions in exon 20. Even if limited by the low number of patients analyzed, erlotinib also demonstrated activity in patients harboring uncommon EGFR mutations. In detail, exon 18 G719 mutations, exon 19 K757R and E746G mutations, exon 20 S768I and exon 21 G836S mutations have been associ- ated with good clinical outcomes, whereas tumors harboring exon 18 S720I mutation had poor responses to erlotinib (Klughammer et al., 2016). Besides this, potential differences in tolerability profile, drugs' interactions and costs are usually taken into account in order to select the best drug for each patient. As regards toxicity, skin rash and diarrhea were the most common adverse events (AEs) reported with all EGFR-TKIs, with a higher percentage observed with afatinib than erlotinib or gefitinib, while the risk of elevated transaminases was higher for gefitinib compared to the other TKIs. The percentage of grade (G) 3-4 AEs was higher with erlotinib (54.1\%), than afatinib (42.1\%) or gefitinib (29.1\%), while no differences in drug-related AEs discontinuation has been observed between the different TKIs (Ding et al., 2016). Furthermore the safety profiles of all three EGFRTKIs became similar if evaluated after an immediate and proactive management of emerging toxicities (Passaro et al., 2014). A posthoc analysis including about 500 patients included in both LL3 and LL6 studies reported a dose-reduction of afatinib from $40 \mathrm{mg}$ to $30 \mathrm{mg}$ or from $30 \mathrm{mg}$ to $20 \mathrm{mg}$ in about $50 \%$ and $30 \%$ of patients respectively, usually occurring within 6 months of TKI therapy. The dose-adjustment was associated with a significant decrease of both all grade and G3-4 AEs, without any influence on treatment benefit (Yang et al., 2016a). These evidences support the possibility to modulate the dose of afatinib according to the patients' tolerability during the treatment course, facilitating the clinical management of patients' toxicities, without influencing treatment efficacy.

\subsection{Direct comparisons}

Recently the LuxLung7 (LL7) study first performed a "head to head" comparison between first-generation TKI gefitinib and second-generation TKI afatinib in first-line treatment of EGFRmutated NSCLC. LL7 is a randomized prospective phase IIb study, including three co-primary end-points, such as PFS, time to treatment failure (TTF), and OS. The results of the study have shown a significant superiority of afatinib over gefitinib both in PFS (11 vs 10,9 months; HR: $0.74,95 \% \mathrm{CI}: 0.57-0.95$, p: 0.017$)$ and TTF (13.7 vs 11.5 months; HR: $0.73,95 \% \mathrm{CI}$ : 0.58-0.92, p: 0.007), regardless from the specific type of EGFR activating mutation (Del19 vs L858R) (Park et al., 2016a). A significant increment of RR has been also reported with afatinib (73\% vs 56\%, p: 0.002). However it has not been translated into an OS benefit. Indeed at a median follow-up of 42.6 months only a trend toward a better OS has been observed in patients receiving afatinib (27.9 vs 24.5 months) which did not reach statistical significance (HR: 0.86, 95\%CI: 0.66-1.12) (Paz-Ares et al., 2017b). Even if OS data are still immature, LL7 can be formally defined as a negative trial. However we should take into account that such study has met two (PFS and TTF) of the three co-primary endpoints, overall showing a significant reduction of $25 \%$ in the risk of $P D$, with about $1 / 4$ of patients reaching a significant PFS benefit $>6$ months with afatinib. Both TTF and PFS as well as OS data reported in LL7 are similar and consistent to those observed in other randomized studies of afatinib in first-line (Sequist et al., 2013; Wu et al., 2014), suggesting a potential superiority of this drug. Furthermore we can't exclude that final OS data, including also late censored events, might reach the statistical significance, definitively confirming the benefit observed in both PFS and TTF, which usually are more informative about first-line treatment efficacy. As regards safety, both drugs were well tolerated with an overall incidence of $\mathrm{G}>3$ AEs of $30 \%$ with afatinib and $20 \%$ with gefitinib, and very low percentages (6\%) of treatment discontinuations due to drug-related AEs in both treatment arms. A significant higher rate of diarrhea (90\%) and stomatitis (64\%) have been reported with afatinib, while transaminases increase was more frequent with gefitinib (24\%). Furthermore about 4 cases of severe interstitial lung disease have been described in gefitinib arm (Park et al., 2016a). ARCHER-1050, NCT01774721, is another ongoing study compar- 
ing first vs second-generation EGFR-TKI (gefitinib vs dacomitinib), but results are not available yet. CTONG 0901 is a phase II study of erlotinib vs gefitinib which didn't show any significant survival differences between the two treatment arms (Yang et al., 2015b). Finally the impressive efficacy results observed with the thirdgeneration EGFR-TKI osimertinib in the first-line cohort of AURA study (RR: 77\%, PFS: 19.7 months) (Ramalingam et al., 2016) have led to the design of the randomized phase III FLAURA trial. This is the first study comparing third-generation TKI osimertinib vs first-generation TKI gefitinib or erlotinib as first-line treatment of EGFR-mutated NSCLC patients, and the results of such trial are eagerly awaited because they could further modify the current treatment algorithm in this subgroup of patients. To date all evidences available about EGFR-TKIs' efficacy and tolerability should be carefully evaluated by clinicians in order to select the best drug for each patients in everyday clinical practice.

\subsection{Activity against brain metastasis}

Brain metastasis (BM) are a common event in EGFR-mutated NSCLC, with major negative impact on patients' QoL and survival, ranging from 4.5 months to 11.0 months after diagnosis (Liao et al., 2015; Umemura et al., 2012). Current treatment options available for EGFR-mutated patients with BM are very limited, also because their participation in prospective clinical trials was historically very low. Stereotactic radiosurgery (SRS) and whole brain radiotherapy (WBRT) concomitantly or followed by EGFR-TKIs remain the current gold standard for patients with BM, even if they are often associated with considerable adverse neurotoxicity and questionable efficacy. Activity of upfront first-generation EGFR-TKIs has been reported in retrospective series including low number of EastAsian patients (Park et al., 2012; Iuchi et al., 2013; Gerber et al., 2014; Zhang et al., 2016a), overall showing poor response rates likely due to the low penetration of such agents across the bloodbrain barrier (BBB) (Zhao et al., 2013; de Vries et al., 2012). Some studies showed that erlotinib has a better central nervous system (CNS) penetration than gefitinib (Togashi et al., 2012), thus suggesting that it could be preferred if TKI monotherapy is used upfront for asymptomatic patients with BM. However a retrospective multi-Institutional analysis has recently demonstrated that the use of upfront EGFR-TKI and deferral of radiotherapy is associated with inferior OS, suggesting that radiotherapy followed by TKI should be considered as the preferred strategy (Magnuson et al., 2017). Encouraging data emerged from the phase I BLOOM study including EGFR-mutated NSCLC patients progressed on prior treatment with EGFR-TKI with a confirmed diagnosis of BM. Among the 21 patients receiving osimertinib $160 \mathrm{mg}$ daily, 33\% had PR and $43 \%$ had SD, with a tolerable safety profile including only $14 \% \mathrm{G} \geq 3$ drug-related AEs (Yang et al., 2016b). Waiting for the results in the T790M-positive cohort included in the BLOOM study, osimertinib has already shown high activity in patients with CNS disease harboring T790M mutation enrolled in two phase II studies (Goss, 2017), likely due to its greater penetration of animal models' BBB compared to other TKIs, gefitinib, rociletinib, or afatinib (Ballard et al., 2016). Furthermore the recent results of the AURA 3 trial demonstrated a significant survival benefit of osimertinib over platinum-chemotherapy in 144 T790M-positive patients with CNS metastasis at baseline, suggesting it as a very effective drug for these patients (Mok et al., 2017). Recently the results of the first prospective randomized trial comparing EGFR-TKI icotinib vs WBRT \pm chemotherapy in EGFR-mutated asian NSCLC patients with $\mathrm{BM}$, have shown a significant superiority of TKI in terms of both intracranial and systemic RR, as well as intracranial and systemic PFS, together with a lower incidence of severe treatment-related AEs (Wu et al., 2017). These data suggest icotinib as new standard 1-line treatment for EGFR-positive asian patients with BM. How- ever data on OS, neurotoxicity and secondary use of WBRT are not available yet. Furthermore it will be useful to see the results of other two ongoing randomized phase III trials comparing upfront EGFRTKI erlotinib or gefitinib vs WBRT (NCT02714010; NCT02338011) including also caucasian population, as well as a potential comparison between third-generation TKI osimertinib vs WBRT, before to modify current treatment recommendations in this setting of patients.

\section{Which therapy can be combined with EGFR-TKIs to improve patients' outcomes?}

\subsection{Anti-angiogenic agents}

Pre-clinical studies have shown a close biological link between EGFR and angiogenetic pathways, likely mediated by signal transducer and activator of transcription 3 (STAT3), which plays a crucial role in the tumor angiogenesis (Hall et al., 2015). A recent study demonstrated that EGFR-mutated NSCLC cell-lines and tissues are associated with increased expression of VEGF and that EGFR-inhibition reduces VEGF levels (Hung et al., 2016). Recently the addition of bevacizumab to the EGFR-TKI, Erlotinib, has been investigated in a phase II randomized study including untreated, EGFR-mutated, East-Asian NSCLC patients. The results of such study showed a significant longer median PFS (16 vs 9.7 months), with about $50 \%$ reduction of the risk of progression (HR: 0.54 (95\% CI: 0.36-0.79)) in favour of the combination arm (Seto et al., 2014). Interestingly the preliminary results of the European BELIEF trial have recently revealed that patients with EGFR-T790M positive tumors would benefit more from erlotinib plus bevacizumab combination with a median PFS of 16 months compared to 10.5 months of patients without T790M, suggesting a new potential strategy to overcome T790M-mediated acquired resistance (Stahel et al., 2015). The major concern for clinical use is represented by the cumulative toxicity, whit about $60 \%$ of G3-4 hypertension and $8 \%$ of G3-4 proteinuria reported in the combination arm, while no differences in drug-discontinuation rate have been observed between the two treatments. On the basis of these positive results erlotinib plus bevacizumab has recently received the approval in first-line by both Food and Drug Administration (FDA) and European Medical Agency (EMA), emerging as new frontline backbone for EGFRmutated NSCLC patients. Although these data are very promising, we would like to see the results of the confirmatory ongoing phase III NEJ026 (Maemondo et al., 2016) and BEVERLY (Gridelli et al., 2016) studies, including Asian and Caucasian patients respectively, before to start using this combination in clinical practice. Similarly the phase III RELAY trial (NCT02411448) is currently investigating ramucirumab in combination with erlotinib vs erlotinib alone as first-line treatment in about 400 EGFR mutated NSCLC patients, but we expect final results that will be available within the two next years.

\subsection{Chemotherapy}

Several phase III studies evaluated the addition of chemotherapy to EGFR-TKIs in first-line (Gatzemeier et al., 2007; Giaccone et al., 2004; Herbst et al., 2004; Herbst et al., 2005; Jänne et al., 2012), demonstrating no survival benefit with combinations, likely because wild-type patients were also enrolled. The FASTACT2 randomized phase III study subsequently showed that the significant improvement of survival outcomes obtained with a first-line intercalated regimen of chemotherapy and erlotinib combination was only limited to the subgroup of patients with an activating EGFRmutation (Wu et al., 2013). 
Recently Ying Cheng and colleagues reported the results of a phase II randomized trial comparing pemetrexed plus gefitinib vs gefitinib in treatment-naive, East Asian patients, with advanced NSCLC and activating EGFR mutations. The study met its primary end-point in the intent-to-treat population, showing a significantly longer median PFS in favour of the combination arm (15.8 vs 10.9 months; HR: 0.68; 95\%CI: 0.48--0.96), independently from the specific type of mutation (EGFR exon 19 deletion vs L858R mutation), while no differences in response rate (RR: $80 \%$ vs $74 \%$ ) were observed between the two treatment arms. As attended, the percentage of patients who reported G3-4 drug-related AEs was significantly higher ( $42 \%$ vs $19 \%$ ) in the combination arm as well as the proportion of patients who discontinued treatment (Cheng et al., 2016). Similarly the NEJ005 randomized phase II study, prospectively compared concurrent gefitinib plus carboplatin/pemetrexed regimen vs sequential alternating regimen in East-Asian, EGFR-mutated NSCLC patients. The results of such study showed a favorable trend in PFS (18.3 vs 15.3 months; HR $0.71,95 \%$ CI 0.42-1.20; $\mathrm{P}=0.20$ ) and a significant improvement in OS (41.9 vs 30.7 months; HR 0.51, 95\% CI 0.26-0.99; P=0.042), in favour of the concurrent regimen arm (Sugawara et al., 2015), demonstrating the superiority of the upfront combination of TKI plus chemo, which is currently investigated in the ongoing phase III study. Both such trials suggested that adding chemotherapy to EGFR-TKI in firstline may improve outcomes of EGFR-mutated patients. The benefit obtained could be likely related to the activity of early concurrent use of cytotoxic agents against de-novo resistance alterations, but the lack of tissue samples collection for biomarker analysis, limited the possibility to evaluate molecular data. The results are intriguing, but need to be confirmed by prospective phase III ongoing studies, including both Asian and Caucasian populations, in order to evaluate the optimal treatment sequence, and ultimately accept the increased adverse events and cost of an upfront combination.

\subsection{Immunotherapy}

Constitutive oncogenic signaling through the EGFR pathway promotes programmed death ligand 1 (PDL1) expression and treatment with PD1 inhibitors may enhance tumor responses in EGFR-mutated pre-clinical models (Akbay et al., 2013; Chen et al., 2015). However the majority of clinical trials of immunecheckpoint inhibitors (ICI) in lung cancer have shown that single agent ICIs are not effective in pre-treated NSCLC patients harboring EGFR-mutations, discouraging their clinical use in this subgroup of patients. A recent meta-analysis also confirmed that PD1/PDL1 inhibitors don't improve OS compared to docetaxel in pre-treated, EGFR-mutated NSCLC patients (Lee et al., 2017 Feb). Translational studies clearly demonstrated that NSCLC with oncogene drivers, including both EGFR activating and resistant mutations, are characterized by a very low tumor mutation burden (TMB), which is commonly considered as good predictor of response to ICI therapy (Spigel et al., 2016; Rizvi et al., 2015). Even if EGFR-mutated tumors are often associated with PDL1 overexpression (D'Incecco et al., 2015), the low rate of tumor infiltrate lymphocytes (TILs) suggests a very low immune-dependence of these tumors. Recently Gainor et al. have found a concurrent PD-L1 overexpression and high rate of TILs in the tumor microenvironment of only 1/57 TKI-naive and 5/57 TKI- resistant patients with EGFR-mutated NSCLC (Gainor et al., 2016), confirming a lack of immunogenicity in the majority of such tumors. Even if PD-L1 expression levels changed in 16 patients evaluated after EGFR-TKI therapy, however not significant variations in both TILs and PDL1 expression have been found before and after TKI. Despite these translational evidences, the results of the phase II BIRCH study have recently demonstrated a great activity of atezolizumab monotherapy as first-line treatment in PDL1-positive NSCLC patients, regardless of tumor EGFR-mutation
status(Garassino et al., 2017). Several trials are currently investigating the potential benefit of PD1/PDL1 ICIs in combination with EGFR-TKI in first-line setting (NCT 02039674, NCT02013219, NCT02143466). Erlotinib plus nivolumab combination resulted in a tolerable safety profile, with durable responses observed in 4/20 EGFR-mutated, TKI-naïve NSCLC patients(Gettinger, 2014). A phase III study of osimertinib plus durvalumab has been prematurely closed because of the high rate of pulmonary toxicities observed with such combination (NCT 02454933). Finally preliminary results of the CheckMate 012 study have recently shown a partial response (PR) in 4/8 EGFR-mutated patients receiving nivolumab plus ipilimumab combination (Hellmann et al., 2016). As suggested by all these translational and clinical data, more efforts are needed in order to clarify the complex interaction between EGFR molecular pathway and anti-tumor immune response, and ultimately select those patients who may benefit from these combinations.

\section{How to overcome acquired resistance to EGFR-TKIs?}

\subsection{EGFR-TKI beyond PD}

Until one year ago, when radiological progression was detected, all the international guidelines recommended to continue the ongoing EGFR-TKI beyond progression disease (PD), with or without loco-regional therapies, except for symptomatic, systemic PD, which required the immediate switching to platinumchemotherapy. The single arm phase II "ASPIRATION" study has recently demonstrated that continuing erlotinib beyond the radiological PD, would benefit a subgroup of patients with limited and asymptomatic disease. Particularly $52 \%$ of patients clinically selected by investigators were able to continue TKI after PD, thus resulting in a 3.1 months longer PFS compared to those who immediately stopped the EGFR-TKI (Park et al., 2016b). Such study, even if limited by several inherent selection biases, confirmed the results emerging from several retrospective series, that continuing TKI beyond radiological PD may represent a reasonable option in a clinically selected subgroup of patients. However who are the "selected patients" who may really benefit from this strategy remains still questionable. Furthermore the results of another phase III randomized "IMPRESS" study failed to demonstrate any survival benefit from continuing gefitinib beyond PD together with platinum-chemotherapy compared to switching to chemotherapy alone. There was no difference in the primary endpoint of PFS (PFS: 5.4 vs 5.4 months; HR: $0.86,95 \% \mathrm{CI}$ : 0.65-1.13), while continuing TKI resulted in a detrimental effect on OS (OS: 14.8 vs 17 months; HR: 1.62, 95\%CI: 1.05-2.52) (Soria et al., 2015). A further analysis of survival according to the T790M circulating tumor (ct) DNA status assessed at baseline revealed that patients without T790M could benefit from combination treatment (PFS: 6.7 vs 5.4; HR: $0.67,95 \% \mathrm{CI}$ : 0.43-1.03). However this positive trend in PFS was not associated to a significant OS improvement, showing HR 1.49 for T790M-positive and HR 1.15 for T790M-negative patients (Soria et al., 2016). Overall these data suggest that patients who progressed to prior EGFR-TKI should not continue TKI beyond PD together with platinum-chemo, because of detrimental effects observed at least in the T790M positive population, while not definitive conclusions may be drawn for T790M-negative patients.

\subsection{New generation EGFR-TKIs}

The identification of the molecular alterations underlying the development of resistance has led to the development of several targeted inhibitors, which are mostly under investigation in clinical studies (Rolfo et al., 2014). The most common mechanisms of acquired resistance is the secondary T790M point mutation in 
Table 1

Third-generation EGFR-TKIs in clinical development in NSCLC.

\begin{tabular}{|c|c|c|c|c|}
\hline EGFR-TKI & Dose & ORR & Toxicity & Status \\
\hline AZD9291 & $80 \mathrm{mg}$ QD & $61 \%$ & Diarrhea, rash, nausea, ILD & Approved \\
\hline CO-1686 & 500 mg BID & $45 \%$ & Hyperglicemia, nausea, diarrhea & Stopped \\
\hline HM61713 & $800 \mathrm{mg}$ QD & $43 \%$ & Diarrhea, rash, nausea, dry skin & Stopped \\
\hline EGF816 & 320 mg/day & $44 \%$ & Rash, Diarrhea, stomatitis, pruritus & Phase II \\
\hline ASP 8273 & $300 \mathrm{mg}$ QD & $38 \%$ & Diarrhea, nausea, vomiting, rash, ILD & Phase II \\
\hline AC 0010 & 250 mg BID & $62 \%$ & Diarrhea, ALT/ASTrash, pruritus, nausea & Phase I \\
\hline
\end{tabular}

exon 20 of EGFR gene, described in about $50-60 \%$ of cases, followed by cMET amplification (5-15\%), Her2 mutations (3-12\%), small cell lung cancer transformation (3-10\%), and others. Particularly, T790M gatekeeper mutation induces both steric hindrance and increased binding affinity for adenosine triphosphate (ATP), which ultimately results in the reduction of both binding and potency of reversible EGFR-TKIs. The understanding of such molecular networks accelerated the development of third-generation, irreversible TKIs, which thanks to their peculiar pyrimidine-based structure, are able to target and inhibit not only EGFR activating mutations, but also the resistant T790M mutation (Socinski et al., 2016; Jia et al., 2016). The advent of these drugs in clinical setting produced a paradigm shift in the management of lung cancer patients who progressed to prior first/second-generation TKIs. A very promising activity and a tolerable safety profile of such compounds emerged from the phase I studies including patients with EGFR-mutated NSCLC who progressed to first-line EGFR-TKI and were T790M positive (Table 1). Osimertinib (AZD9291) represents the compound in most advanced stage of clinical development. The AURA1 study has first shown an impressive ORR: 60\%, disease control rate (DCR): 95\%, PFS: 9.6 months with minimal side effects in the subgroup of patients with 790M mutation(Skoulidis and Papadimitrakopoulou, 2017), leading to the fast-track approval by regulatory authorities for the treatment of patients who develop resistance to first-generation TKIs and were T790 M positive. The results of the phase III randomized AURA3 trial, comparing osimertinib vs platinum-pemetrexed chemotherapy in this setting of patients have been recently reported at the last World Conference on Lung Cancer (WCLC) 2016 and simultaneously published on the New England Journal of Medicine (NEJM) (Mok et al., 2017). The study met its primary endpoint showing a significant longer median PFS in patients receiving osimertinib compared to platinum chemotherapy (PFS: 10.1 vs 4.4 months; HR: 0.30 , 95\%CI: $0.23-0.41$ ), including also the subgroup of patients with brain metastasis (HR: $0.32,95 \% \mathrm{CI}$ : $0.21-0.49$ ). RR were significantly higher (71\% vs $31 \%$ ), as well as more durable (DoR: 9.7 vs 4.1 months) with osimertinib compared to standard chemotherapy. As regards the safety profile osimertinib was associated with lower rates of G3-4 AEs compared to platinum-pemetrexed. These impressive results confirm that osimertinib is the new standard of care in patients with EGFR-T790 M positive NSCLC who failed firstline EGFR-TKI treatment. Indeed all the international guidelines have already incorporated osimertinib as recommended option in this setting. As regards the other new-generation TKIs, unfortunately the clinical development of both rociletinib and olmutinib has been recently stopped, even if both these agents had already received the FDA-break-through therapy designation in lung cancer. Despite the promising activity observed in phase I study (Jänne et al., 2015), the updated "confirmed" response rate to rociletinib dropped from $59 \%$ to $39 \%$ at $625 \mathrm{mg}$ bid dose $(\mathrm{n}=175)$ and to $25 \%$ at $500 \mathrm{mg}$ bid dose $(\mathrm{n}=156)$ in T790M-positive patients (Sequist et al., 2015; Goldman et al., 2016). These data, along with the high rate of G3-4 hyperglycemia and QTc prolongation reported in TIGERX trial, led to the FDA notification with the subsequent stopping of patients' enrollment in all ongoing trials with rociletinib. Other third-generation TKIs in clinical development include
EGF816, ASP8273, and AC0010, all showing a great activity and a tolerable safety profile in phase I studies (Yu et al., 2016; Tan et al., 2016; Zhang et al., 2016b). However as learned by rociletinib history, a careful evaluation of the results obtained from the small datasets of early clinical trials is recommended and larger phase II-III ongoing trials are needed to confirm these preliminary observations.

\section{Which is the current role of liquid biopsy?}

\subsection{Potential application at diagnosis}

The EGFR mutation testing of tumor samples DNA at diagnosis is recommended by all the international guidelines for advanced nonsquamous NSCLC in order to decide the proper therapeutic strategy for each patient. Even if tissue biopsy remains the current goldstandard, however it's limited by several features, such as the not easy access to different tumor sites, the invasiveness of procedures, the tumor heterogeneity, and not ultimately the low patients' compliance. Thus, in the last decade an alternative not invasive approach, known as liquid biopsy, has been proposed to overcome the aforementioned issues. An increasing number of studies and meta-analysis evaluated the diagnostic value of plasma-based EGFR testing in the management of NSCLC patients, overall showing a sensitivity of 0.62 and a specificity of 0.96 as compared with the standard tissue genotyping, which suggest an adequate diagnostic accuracy of circulating tumor (ct)DNA analysis (Qiu et al., 2015; Wu et al., 2015; Qian et al., 2016; Luo et al., 2014). These evidences have led to the analytical validation and the clinical approval of EGFR mutation testing by using ctDNA isolated from plasma or serum of about $30 \%$ of patients whose tissue is not available at diagnosis or tissue analysis results are not evaluable (Fig. 1). However recent data have suggested that the ability to identify EGFR activating mutations in plasma of NSCLC patients is significantly higher in the presence of extra-thoracic (M1b) vs intra-thoracic (M1a) disease, and it should be taken into account in the clinical management of our patients (Karlovich et al., 2016; Reck et al., 2016). In the study of Sacher et al. plasma genotyping by droplet digital PCR (ddPCR) demonstrated a very high specificity (100\%) and sensitivity (74-82\%) in 80 patients with advanced NSCLC harboring activating EGFR del19/L858R mutations (Sacher et al., 2016). The open-label IFUM trial showed a high concordance rate of $94 \%$ between plasmabased (ctDNA) EGFR mutation testing and tissue-based testing in about 650 patients with EGFR-positive advanced NSCLC (Douillard et al., 2014). The promising diagnostic performance of ctDNA seen in this controlled trials was subsequently confirmed in a real world diagnostic setting. The multicenter ASSESS study has recently demonstrated a similar concordance rate of $89 \%$ (sensitivity $46 \%$, specificity 97\%) between these two methods in detecting the EGFR mutation status in 1162 patients with advanced NSCLC, suggesting the clinical utility and reliability of ctDNA for EGFR mutation analysis at diagnosis (Reck et al., 2016). However some efforts are needed to raise the sensitivity of this approach. Despite Real-Time (RT)-PCR and droplet digital (dd)PCR technologies are most commonly used for ctDNA analysis in clinical studies, next generation sequencing (NGS) is emerging as a very promising tool that can 


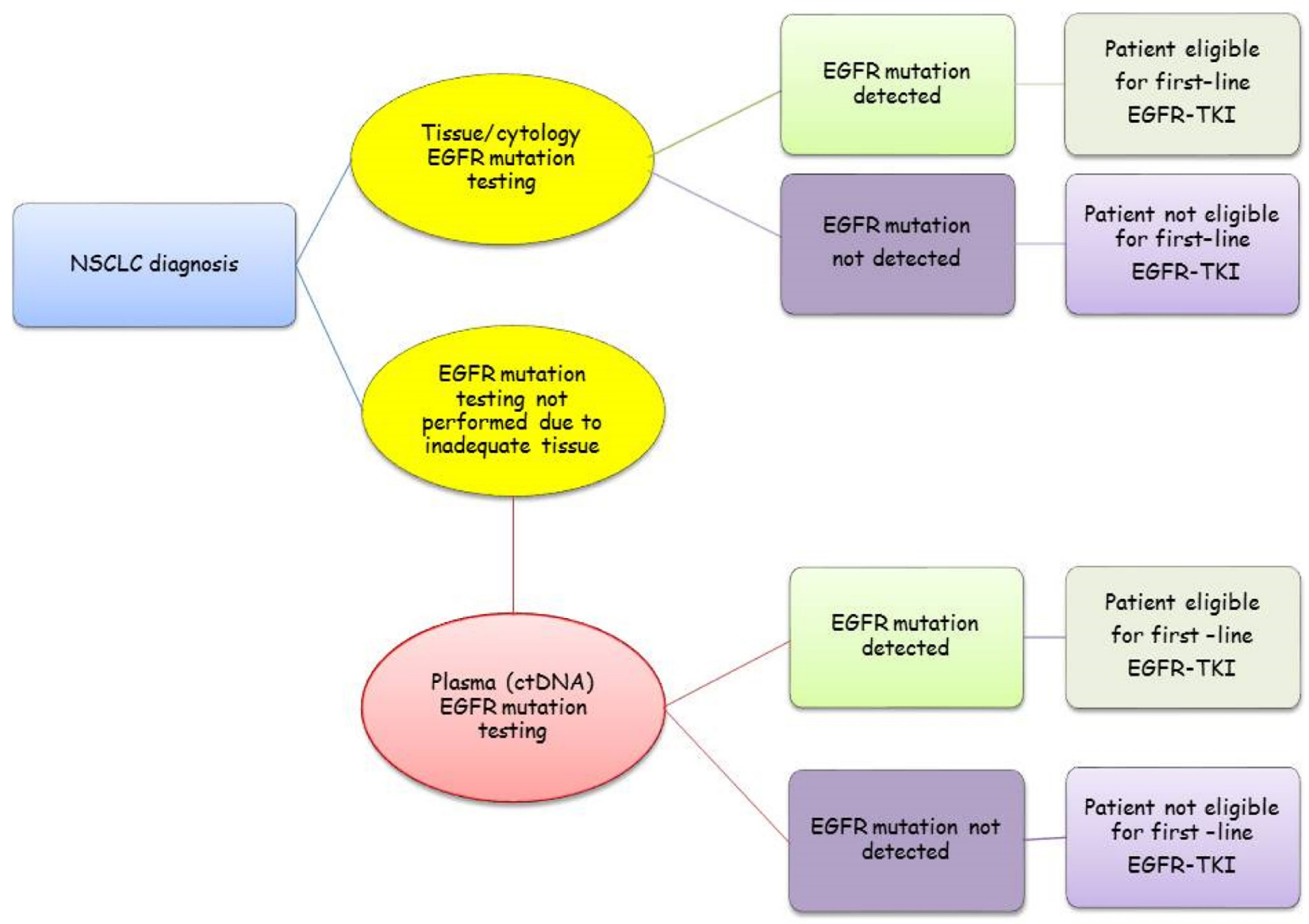

Fig. 1. The role of plasma (ctDNA) EGFR mutation testing at the time of NSCLC diagnosis.

allow to overcome several limitations inherent to the other techniques. Indeed there are several commercially available NGS panels specifically designed for ctDNA testing in lung cancer, which are currently under evaluation in clinical studies. Recently Villaflor et al. assessed the utility of two ctDNA panels in a clinical series of 68 NSCLC patients; the 54-gene panel include only mutations whereas the 68-gene include also ALK, RET or ROS1 fusions. In this paper it was also investigated the concordance between paired tissue and blood samples whenever possible. The results showed that $80 \%$ of patients have detectable ctDNA, with $83 \%$ presenting at least one non-synonymous ctDNA alteration. As expected the most frequent mutations are reported in TP53, KRAS and EGFR genes. Nevertheless $17 \%$ of patients had no ctDNA alterations, even if for 5 of them an oncogenic driver mutation has been identified by tissue-based analysis (Villaflor et al., 2016). Therefore we have to be aware that liquid biopsy analysis may not always identify driver mutations. Another recent paper evaluated NGS to characterize 112 plasma samples from 102 patients with advanced NSCLC, detecting 275 alterations in 45 genes in $84 \%$ of patients (86 of 102). As well as reported in the paper from Villaflor (Villaflor et al., 2016), NGS was able to detect mutations in additional genes for which experimental drugs were available in clinical trials. The concordance between tissue and plasma was 79\%; interestingly it increased when a shorter time interval between tissue and blood collection was reported (Thompson et al., 2016). Finally ct-DNA analysis by NGS of over 5000 patients with advanced NSCLC identified EGFR mutations in about $25 \%$ of patients at baseline, thus highly consistent with the mutation rate reported by the cancer genome atlas (TCGA) tissue-based analysis. Furthermore additional driver mutations were identified in about $38 \%$ of patients whose tumor tissue genotyping failed (Mack et al., 2017). Another study showed a high concordance rate of $88 \%$ between urine ctDNA and tissue genotyping on EGFR-mutation status detection at baseline, revealing a strong correlation with patients' outcomes during EGFR-TKI therapy (Chen et al., 2017). However a standardization and a prospective validation of ctDNA methodology are required before its widespread clinical application.

\subsection{Potential application at progression}

Recently a growing interest has been focused also on monitoring dynamic changes in plasma of both sensitizing and resistant EGFR-mutations during TKI treatment. Particularly after the clinical approval of osimertinib in United States, Europe and Japan, re-biopsy at progression became mandatory, in order to identify T790M mutation or other alternative mechanisms of acquired resistance and ultimately personalize second-line therapy. Singlecenter real-world series demonstrated that repeating tissue biopsy at the time of PD was feasible in about $80 \%$ of patients with more than $20 \%$ of them having inadequate material for mutation testing and only $30 \%$ of patients benefiting from such procedure (Yoon et al., 2012; Chouaid et al., 2014). The non-invasive potential of ctDNA has been deeply studied by Oxnard in many studies. In 2014, he first demonstrated that the analysis of ctDNA through ddPCR in serial plasma sampling during TKI treatment, allowed the detection of the resistance T790M mutation weeks and sometimes months prior to radiological PD, highlighting the possibility to anticipate clinical evidence of progression through early molecular evidences 


\section{Tissue-based paradigm}

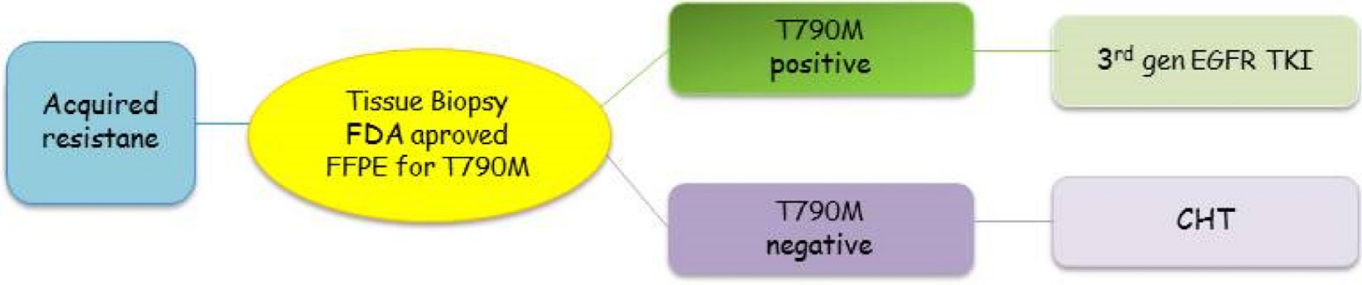

\section{Plasma-based paradigm}

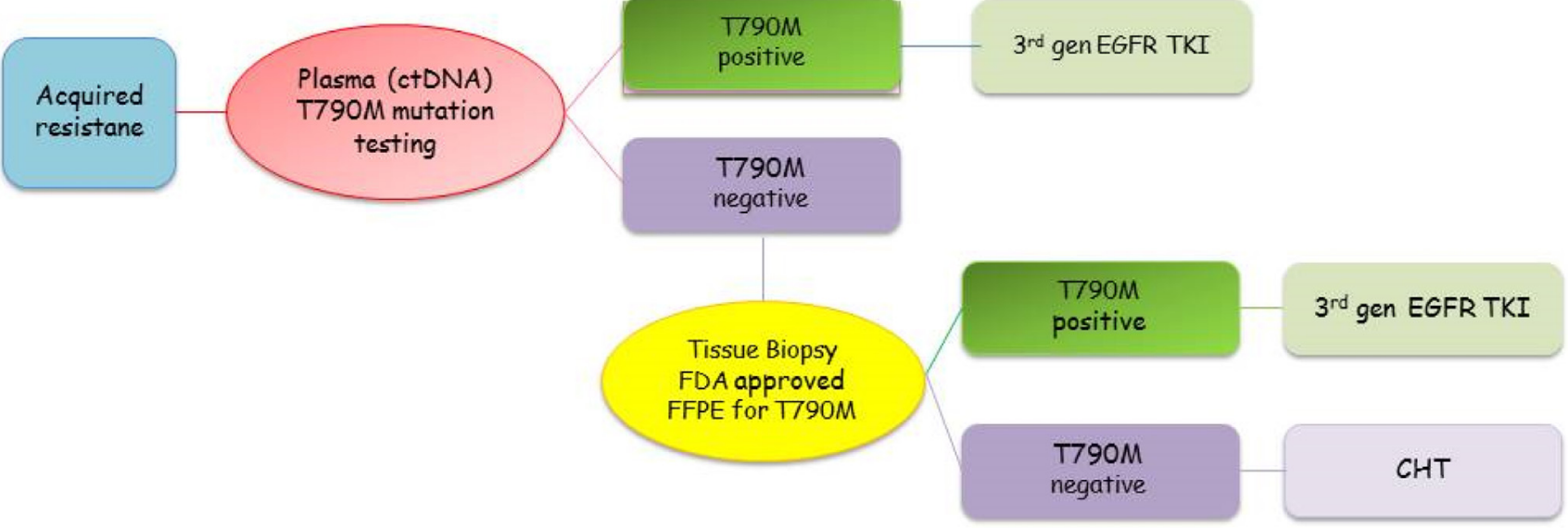

Fig. 2. The role of plasma (ctDNA) EGFR-T790M mutation testing at the time of NSCLC progression.

(Oxnard et al., 2014). Recently his group prospectively evaluated the sensitivity and specificity of plasma genotyping by ddPCR in 180 patients with advanced NSCLC, including 60 patients with acquired resistance to EGFR-TKI. Plasma genotyping by ddPCR exhibited $79 \%$ specificity and $77 \%$ sensitivity in the detection of T790M mutation, which are lower than those observed with EGFR-activating mutations at baseline (Oxnard et al., 2016). In addition Oxnard et al. showed that outcomes of T790M-positive patients included in the phase I AURA study were similar if T790M was detected in plasma or tumor tissue. Conversely both RR and PFS of T790M-negative patients on plasma were significantly higher than T790M-negative on tissue, and further tumor genotyping of plasma T790M-negative patients allowed to identify a subgroup of T790M-positive patients on tumor tissues who had better outcomes (Oxnard et al., 2016). According to these data the authors suggest that plasma genotyping could represent the first step for the detection of T790M status at the time of PD. However, because of the low sensitivity (70\%) of the current available technologies which are associated with a $30 \%$ false negative rate, patients with T790M-negative on plasma should repeat tumor tissue biopsy to further investigate the presence of such molecular alteration (Fig. 2). Interestingly, 18/164 patients with plasma T790M-positive genotyping were T790Mnegative on tissue analysis, showing favorable clinical outcomes similar to those of patients with T790M-positive tumor tissue (Oxnard et al., 2016). These data, consistently with those observed in other studies evaluating plasma genotyping approach at the time of PD (Takahama et al., 2016; Thress et al., 2015), demonstrated a lower specificity of plasma-based detection of T790M at PD compared to EGFR-activating mutations detection at baseline, likely due to the higher heterogeneity of TKI-resistant tumors. As single tissue biopsy is just a snapshot of the tumor not reflecting its spatial heterogeneity, ctDNA could be more representative of the overall tumor mutation status, allowing to identify different targetable alterations driving tumor resistance. These suggestions have been recently confirmed by a paper of Chabon et al., who performed CAPP-Seq ctDNA profiling on 41 patients harboring both EGFR-activating and resistant T790M mutations on tumor tissue after progression to prior EGFR TKI therapy. The CAPP-Seq ctDNA analysis confirmed a single T790M mutation in $54 \%$ of patients, while revealed additional molecular alterations in $46 \%$ of them, including 34\% MET or HER2 increased gene copy number (GCN), $7 \%$ single nucleotide variations (SNVs) in EGFR, PIK3CA, or Rb1, and $5 \%$ both MET GCN and PIK3CA or Rb1 SNVs (Chabon et al., 2016). Thus ctDNA genotyping allows to more accurately identify the presence of potential multiple mechanisms of resistance emerging during TKI therapy revealing a higher frequency (46\%) of intra-tumor heterogeneity than that (5-15\%) reported in previous studies (Sequist et al., 2011; Yu et al., 2013). Since the co-occurrence of different molecular alterations has been associated with inferior outcomes to subsequent third-generation TKI therapy, ctDNA analysis could represent a useful tool to guide the clinicians in the selection of the best treatment strategy for each patient. In addition to plasma, urine genotyping has also shown a high sensitivity in detecting T790M mutation status, ranging from $72 \%$ to $93 \%$, in preliminary studies including few patients (Reckamp et al., 2016), and is currently under investigation in trials including larger cohorts of patients. Interestingly combined plasma and urine testing identified 12 additional T790M positive patients who were 
negative on tissue and received benefit from Rociletinib therapy, and an early decrease of urine T790M levels was described in 9 patients during such treatment (Reckamp et al., 2016). Notably it has been recently developed a novel targeted NGS approach for the detection of both driver mutations and rearrangements in ctDNA from advanced NSCLC patients. The assay and analysis software were able to identify mutations present at $0.1 \%$, and the diagnostic performance was even better, reaching $100 \%$ sensitivity and specificity, when mutations were present at an allelic frequency $0.4 \%$ or greater (Paweletz et al., 2016). The advent of these highly sensitive technologies offer the opportunity to monitor EGFR-TKI therapy revealing early dynamic changes in plasma of both sensitizing and resistant EGFR-mutations which may anticipate the clinical/radiological PD. However waiting for analytical and clinical validation of ctDNA in disease monitoring within controlled prospective trials, the T790M mutation ctDNA analysis with or without complementary tissue biopsy is currently recommended in all patients who develop radiological PD to prior EGFR-TKI.

\section{Conclusions}

To date we are witnessing a second revolution in the management of EGFR-mutated NSCLC. In this review we have outlined the most important innovations emerging from clinical studies. First and second generation EGFR-TKIs reached a survival plateau ranging from 21 to 30 months OS in first-line, without any significant differences observed in direct comparison trials. The addition of both anti-angiogenic agents and chemotherapy seem to raise the effectiveness of single agent EGFR-TKI, likely thanks to the activity of early concurrent use of such drugs against de-novo resistance alterations, but the preliminary results observed in asian populations need to be confirmed by prospective phase III ongoing studies, including also caucasian patients. Despite poor outcomes observed with single agent ICIs in pre-treated EGFR-mutated patients, an improved understanding of the complex interaction between EGFR molecular pathway and anti-tumor immune response is essential to identify those patients who could benefit from upfront EGFR-TKI plus ICI combinations. Third generation EGFR-inhibitors seem to be very promising drugs, which thanks to their great activity and tolerable safety profile, offer a new potential of cure for patients who failed first-generation EGFR-TKI and are T790M-positive. Osimertinib may be considered the first in class of such new family of compounds, as it has been already approved for clinical use, representing a new available weapon to overcome tumor resistance and ultimately improve patients' survival. Promising data have been observed also in pre-treated patients with BM, but randomized trials in this subset are still ongoing. Results of FLAURA trial will be crucial to confirm the potential benefit of osimertinib in firstline. Following the clinical approval of osimertinib re-biopsy at progression became mandatory, in order to re-analyze the tumor molecular profile and identify T790M mutation. In this scenario ctDNA analysis on plasma samples is currently recommended as first step of tumor genotyping, followed by tissue biopsy only for those patients who are T790M-negative, while EGFR mutation testing by ctDNA at baseline is approved only for a minority of patients whose tissue is not available at diagnosis or tissue analysis results are not evaluable. The intratumor heterogeneity found in resistant NSCLC suggests that, thanks to the development of highly sensitive technologies, ctDNA genotyping will allow to more accurately identify the presence of potential multiple mechanisms of resistance emerging during TKI therapy, guiding the selection of the best treatment strategy for each patient. Very exciting is also the promise of ctDNA in disease monitoring, however there are several issues which need to be elucidated and prospective validation is crucial before clinical application. Considering the advent of new promis- ing drugs/combinations, the main challenge will be how to combine all these agents in order to define the optimal treatment algorithm and ultimately improve the survival outcomes of EGFR-mutated NSCLC patients.

\section{References}

Akbay, E.A., Koyama, S., Carretero, J., et al., 2013. Activation of the PD-1 pathway contributes to immune escape in EGFR-driven lung tumors. Cancer Discov. 3, 1355-1363.

Ballard, P., Yates, J.W., Yang, Z., et al., 2016. Preclinical comparison of osimertinib with other EGFR-TKIs in EGFR-Mutant NSCLC brain metastases models, and early evidence of clinical brain metastases activity. Clin. Cancer Res. 22, $5130-5140$.

Bronte, G., Rolfo, C., Giovannetti, E., et al., 2014. Are erlotinib and gefitinib interchangeable, opposite or complementary for non-small cell lung cancer treatment? Biological, pharmacological and clinical aspects. Crit. Rev. Oncol. Hematol. 89, 300-313.

Chabon, J.J., Simmons, A.D., Lovejoy, A.F., et al., 2016. Corrigendum: circulating tumour DNA profiling reveals heterogeneity of EGFR inhibitor resistance mechanisms in lung cancer patients. Nat. Commun. 7, 13513.

Chen, N., Fang, W., Zhan, J., et al., 2015. Upregulation of PD-L1 by EGFR activation mediates the immune escape in EGFR-Driven NSCLC: implication for optiona immune targeted therapy for NSCLC patients with EGFR mutation. J. Thorac. Oncol. 10, 910-923.

Chen, S., Zhao, J., Cui, L., Liu, Y., 2017. Urinary circulating DNA detection for dynamic tracking of EGFR mutations for NSCLC patients treated with EGFR-TKIs. Clin. Transl. Oncol. 19 (3), 332-340.

Cheng, Y., Murakami, H., Yang, P.C., et al., 2016. Randomized phase II trial of gefitinib with and without pemetrexed as first-line therapy in patients with advanced nonsquamous non-small-cell lung cancer with activating epidermal growth factor receptor mutations. J. Clin. Oncol. 34, 3258-3266.

Chouaid, C., Dujon, C., Do, P., et al., 2014. Feasibility and clinical impact of re-biopsy in advanced non small-cell lung cancer: a prospective multicenter study in a real-world setting (GFPC study 12-01). Lung Cancer 86, 170-173.

de Vries, N.A., Buckle, T., Zhao, J., et al., 2012. Restricted brain penetration of the tyrosine kinase inhibitor erlotinib due to the drug transporters P-gp and BCRP. Invest. New Drugs 30, 443-449.

D'Incecco, A., Andreozzi, M., Ludovini, V., et al., 2015. PD-1 and PD-L1 expression in molecularly selected non-small-cell lung cancer patients. Br. J. Cancer 112 , 95-102.

Ding, P.N., Lord, S.J., Gebski, V., et al., 2016. Risk of treatment-related toxicities from EGFR tyrosine kinase inhibitors: a meta-analysis of clinical trials of gefitinib, erlotinib and afatinib in advanced EGFR-mutated non-small-cell lung cancer. J. Thorac. Oncol. (19), pii: S1556-0864(16)33582-1.

Douillard, J.Y., Ostoros, G., Cobo, M., et al., 2014. Gefitinib treatment in EGFR mutated caucasian NSCLC: circulating-free tumor DNA as a surrogate for determination of EGFR status. J. Thorac. Oncol. 9, 1345-1353.

Gainor, J.F., Shaw, A.T., Sequist, L.V., et al., 2016. EGFR mutations and ALK rearrangements are associated with low response rates to PD-1 pathway blockade in non-small cell lung cancer: a retrospective analysis. Clin. Cancer Res. 22, 4585-4593.

Garassino, M., et al., 2017. Atezolizumab as $1 \mathrm{~L}$ therapy for advanced NSCLC in PD-L1-selected patients: updated ORR, PFS and OS data from the BIRCH study. J. Thorac. Oncol. 12 (1), S6

Gatzemeier, U., Pluzanska, A., Szczesna, A., et al., 2007. Phase III study of erlotinib in combination with cisplatin and gemcitabine in advanced non-small-cell lung cancer: the Tarceva Lung Cancer Investigation Trial. J. Clin. Oncol. 25, 1545-1552.

Gerber, N.K., Yamada, Y., Rimner, A., et al., 2014. Erlotinib versus radiation therapy for brain metastases in patients with EGFR-mutant lung adenocarcinoma. Int. J. Radiat. Oncol. Biol. Phys. 89, 322-329.

Gettinger, S.ea., 2014. Safety and response with nivolumab (anti-PD-1; BMS-936558, ONO- 4538) plus erlotinib in patients (pts) with epidermal growth factor receptor mutant (EGFR MT) advanced non-small cell lung cancer (NSCLC). J. Clin. Oncol. 32, 5s, suppl; abstr 8022.

Giaccone, G., Herbst, R.S., Manegold, C., et al., 2004. Gefitinib in combination with gemcitabine and cisplatin in advanced non-small-cell lung cancer: a phase III trial-INTACT 1. J. Clin. Oncol. 22, 777-784

Goldman, G.W., et al., 2016. Updated results from TIGER-X, a phase I/II open label study of rociletinib in patients (pts) with advanced, recurrent T790M-positive non-small cell lung cancer (NSCLC). Int. J. Clin. Oncol. 34 (Suppl. 15), abstract 9045.

Goss, G., et al., 2017. CNS response to osimertinib in patients with T790M-positive advanced NSCLC: pooled data from two phase II trials. J. Thorac. Oncol. 12 (1), S6.

Gridelli, C., Rossi, A., Ciardiello, F., et al., 2016. BEVERLY: rationale and design of a randomized open-label phase III trial comparing bevacizumab plus erlotinib versus erlotinib alone as first-line treatment of patients with EGFR-Mutated advanced nonsquamous non-small-cell lung cancer. Clin. Lung Cancer 17, 461-465.

Hall, R.D., Le, T.M., Haggstrom, D.E., Gentzler, R.D., 2015. Angiogenesis inhibition as a therapeutic strategy in non-small cell lung cancer (NSCLC). Transl. Lung Cancer Res. 4, 515-523. 
Haspinger, E.R., Agustoni, F., Torri, V., et al., 2015. Is there evidence for different effects among EGFR-TKIs? Systematic review and meta-analysis of EGFR tyrosine kinase inhibitors (TKIs) versus chemotherapy as first-line treatment for patients harboring EGFR mutations. Crit. Rev. Oncol. Hematol. 94, 213-227.

Hellmann, M.D., et al., 2016. CheckMate 012: safety and efficacy of first-line (1L) nivolumab (nivo; N) and ipilimumab (ipi; I) in advanced (adv) NSCLC. J. Clin. Oncol. 34, suppl; abstr 3001.

Herbst, R.S., Giaccone, G., Schiller, J.H., et al., 2004. Gefitinib in combination with paclitaxel and carboplatin in advanced non-small-cell lung cancer: a phase III trial-INTACT 2. J. Clin. Oncol. 22, 785-794.

Herbst, R.S., Prager, D., Hermann, R., et al., 2005. TRIBUTE: a phase III trial of erlotinib hydrochloride (OSI-774) combined with carboplatin and paclitaxel chemotherapy in advanced non-small-cell lung cancer. J. Clin. Oncol. 23, 5892-5899.

Hung, M.S., Chen, I.C., Lin, P.Y., et al., 2016. Epidermal growth factor receptor mutation enhances expression of vascular endothelial growth factor in lung cancer. Oncol. Lett. 12 (December (6)), 4598-4604.

Iuchi, T., Shingyoji, M., Sakaida, T., et al., 2013. Phase II trial of gefitinib alone without radiation therapy for Japanese patients with brain metastases from EGFR-mutant lung adenocarcinoma. Lung Cancer 82, 282-287.

Jänne, P.A., Wang, X., Socinski, M.A., et al., 2012. Randomized phase II trial of erlotinib alone or with carboplatin and paclitaxel in patients who were never or light former smokers with advanced lung adenocarcinoma: CALGB 30406 trial. J. Clin. Oncol. 30, 2063-2069.

Jänne, P.A., Yang, J.C., Kim, D.W., et al., 2015. AZD9291 in EGFR inhibitor-resistant non-small-cell lung cancer. N. Engl. J. Med. 372, 1689-1699.

Jia, Y., Yun, C.H., Park, E., et al., 2016. Overcoming EGFR(T790M) and EGFR(C797S) resistance with mutant-selective allosteric inhibitors. Nature 534, 129-132.

Karlovich, C., Goldman, J.W., Sun, J.M., et al., 2016. Assessment of EGFR mutation status in matched plasma and tumor tissue of NSCLC patients from a phase I study of rociletinib (CO-1686). Clin. Cancer Res. 22 (May (10)), 2386-2395.

Klughammer, B., Brugger, W., Cappuzzo, F., et al., 2016. Examining treatment outcomes with erlotinib in patients with advanced non-small cell lung cancer whose tumors harbor uncommon EGFR mutations. J. Thorac. Oncol. 11, 545-555.

Lee, C.K., Man, J., Lord, S., et al., 2017 Feb. Checkpoint inhibitors in metastatic EGFR-mutated non-small cell lung cancer-a meta-analysis. J. Thorac. Oncol. 12 (2), 403-407.

Liao, B.C., Lee, J.H., Lin, C.C., et al., 2015. Epidermal growth factor receptor tyrosine kinase inhibitors for non-small-cell lung cancer patients with leptomeningeal carcinomatosis. J. Thorac. Oncol. 10, 1754-1761.

Luo, J., Shen, L., Zheng, D., 2014. Diagnostic value of circulating free DNA for the detection of EGFR mutation status in NSCLC: a systematic review and meta-analysis. Sci. Rep. 4, 6269.

Mack, P., et al., 2017. Clinical utility of circulating tumor DNA (ctDNA) analysis by digital next generation sequencing of over 5000 advanced NSCLC patients. J. Thorac. Oncol. 12 (1), S6.

Maemondo, M., Inoue, A., Kobayashi, K., et al., 2010. Gefitinib or chemotherapy for non-small-cell lung cancer with mutated EGFR. N. Engl. J. Med. 362, 2380-2388.

Maemondo, M., et al., 2016. NEJ026: Phase III study comparing bevacizumab plus erlotinib to erlotinib in patients with untreated NSCLC harboring activating EGFR mutations. Ann. Oncol. 27 (Suppl. 6), http://dx.doi.org/10.1093/annonc/ mdw383.86.

Magnuson, W.J., Lester-Coll, N.H., Wu, A.J., et al., 2017. Management of brain metastases in tyrosine kinase inhibitor-naïve epidermal growth factor receptor-mutant non-small-cell lung cancer: a retrospective multi-Institutional analysis. J. Clin. Oncol., JCO2016697144.

Mitsudomi, T., Morita, S., Yatabe, Y., et al., 2010. Gefitinib versus cisplatin plus docetaxel in patients with non-small-cell lung cancer harbouring mutations of the epidermal growth factor receptor (WJTOG3405): an open label, randomised phase 3 trial. Lancet Oncol. 11, 121-128.

Mok, T.S., Wu, Y.L., Ahn, M.J., et al., 2017. Osimertinib or platinum-pemetrexed in EGFR T790M-positive lung cancer. N. Engl. J. Med. 376 (7), 629-640.

Network CGA, 2012. Comprehensive molecular portraits of human breast tumours. Nature 490, 61-70.

Oxnard, G.R., Paweletz, C.P., Kuang, Y., et al., 2014. Noninvasive detection of response and resistance in EGFR-mutant lung cancer using quantitative next-generation genotyping of cell-free plasma DNA. Clin. Cancer Res. 20, $1698-1705$

Oxnard, G.R., Thress, K.S., Alden, R.S., et al., 2016. Association between plasma genotyping and outcomes of treatment with osimertinib (AZD9291) in advanced non-small-cell lung cancer. J. Clin. Oncol. 34, 3375-3382.

Park, S.J., Kim, H.T., Lee, D.H., et al., 2012. Efficacy of epidermal growth factor receptor tyrosine kinase inhibitors for brain metastasis in non-small cell lung cancer patients harboring either exon 19 or 21 mutation. Lung Cancer 77, 556-560.

Park, K., Tan, E.H., O’Byrne, K., et al., 2016a. Afatinib versus gefitinib as first-line treatment of patients with EGFR mutation-positive non-small-cell lung cancer (LUX-Lung 7): a phase 2B, open-label, randomised controlled trial. Lancet Oncol. 17, 577-589.

Park, K., Yu, C.J., Kim, S.W., et al., 2016b. First-line erlotinib therapy until and beyond response evaluation criteria in solid tumors progression in asian patients with epidermal growth factor receptor mutation-positive non-small-cell lung cancer: the ASPIRATION study. JAMA Oncol. 2, 305-312.
Passaro, A., Di Maio, M., Del Signore, E., et al., 2014. management of nonhematologic toxicities associated with different EGFR-TKIs in advanced NSCLC: a comparison analysis. Clin. Lung Cancer 15, 307-312.

Passiglia, F., Bronte, G., Castiglia, M., et al., 2015. Prognostic and predictive biomarkers for targeted therapy in NSCLC: for whom the bell tolls. Expert Opin. Biol. Ther., 1-14.

Paweletz, C.P., Sacher, A.G., Raymond, C.K., et al., 2016. Bias-Corrected targeted next-generation sequencing for rapid, multiplexed detection of actionable alterations in cell-free DNA from advanced lung cancer patients. Clin. Cancer Res. 22, 915-922.

Paz-Ares, L., Tan, E.H., O’Byrne, K., et al., 2017a. Afatinib versus gefitinib in patients with EGFR mutation-positive advanced non-small-cell lung cancer: overall survival data from the phase IIb LUX-Lung 7 trial. Ann. Oncol., http://dx.doi. org/10.1093/annonc/mdw611.

Paz-Ares, L., Tan, E.H., O'Byrne, K., et al., 2017b. Afatinib versus gefitinib in patients with EGFR mutation-positive advanced non-small-cell lung cancer: overall survival data from the phase IIb LUX-Lung 7 trial. Ann. Oncol. (February).

Qian, X., Liu, J., Sun, Y., et al., 2016. Circulating cell-free DNA has a high degree of specificity to detect exon 19 deletions and the single-point substitution mutation L858R in non-small cell lung cancer. Oncotarget 7, 29154-29165.

Qiu, M., Wang, J., Xu, Y., et al., 2015. Circulating tumor DNA is effective for the detection of EGFR mutation in non-small cell lung cancer: a meta-analysis Cancer Epidemiol. Biomark. Prev. 24, 206-212.

Ramalingam, S., Yang, J.C., Lee, C.K., et al., 2016. LBA1_PR: Osimertinib as first-line treatment for EGFR mutation-positive advanced NSCLC: updated efficacy and safety results from two Phase I expansion cohorts. J. Thorac. Oncol. 11, S152.

Reck, M., Hagiwara, K., Han, B., et al., 2016. ctDNA determination of EGFR mutation status in european and japanese patients with advanced NSCLC: the ASSESS study. J. Thorac. Oncol. 11 (October (10)), 1682-1689.

Reckamp, K.L., Melnikova, V.O., Karlovich, C., et al., 2016. A highly sensitive and quantitative test platform for detection of NSCLC EGFR mutations in urine and plasma. J. Thorac. Oncol. 11, 1690-1700.

Reis-Filho, J.S., Pusztai, L., 2011. Gene expression profiling in breast cancer: classification, prognostication, and prediction. Lancet 378, 1812-1823.

Rizvi, N.A., Hellmann, M.D., Snyder, A., et al., 2015. Cancer immunology: mutational landscape determines sensitivity to PD-1 blockade in non-small cell lung cancer. Science 348, 124-128.

Rolfo, C., Giovannetti, E., Hong, D.S., et al., 2014. Novel therapeutic strategies for patients with NSCLC that do not respond to treatment with EGFR inhibitors. Cancer Treat. Rev. 40, 990-1004.

Sacher, A.G., Paweletz, C., Dahlberg, S.E., et al., 2016. Prospective validation of rapid plasma genotyping for the detection of EGFR and KRAS mutations in advanced lung cancer. JAMA Oncol. 2, 1014-1022.

Sequist, L.V., Waltman, B.A., Dias-Santagata, D., et al., 2011. Genotypic and histological evolution of lung cancers acquiring resistance to EGFR inhibitors. Sci. Transl. Med. 3, 75ra26.

Sequist, L.V., Yang, J.C., Yamamoto, N., et al., 2013. Phase III study of afatinib or cisplatin plus pemetrexed in patients with metastatic lung adenocarcinoma with EGFR mutations. J. Clin. Oncol. 31, 3327-3334.

Sequist, L.V., Soria, J.C., Goldman, J.W., et al., 2015. Rociletinib in EGFR-mutated non-small-cell lung cancer. N. Engl. J. Med. 372, 1700-1709.

Seto, T., Kato, T., Nishio, M., et al., 2014. Erlotinib alone or with bevacizumab as first-line therapy in patients with advanced non-squamous non-small-cell lung cancer harbouring EGFR mutations (JO25567): an open-label, randomised, multicentre, phase 2 study. Lancet Oncol. 15, 1236-1244.

Skoulidis, F., Papadimitrakopoulou, V.A., 2017. Targeting the gatekeeper: osimertinib in EGFR T790M mutation-positive non-small cell lung cancer. Clin. Cancer Res. 23 (3), 618-622.

Socinski, M.A., Villaruz, L.C., Ross, J., 2016. Understanding mechanisms of resistance in the epithelial growth factor receptor in non-small cell lung cancer and the role of biopsy at progression. Oncologist 22 (1), 3-11.

Soria, J.C., Wu, Y.L., Nakagawa, K., et al., 2015. Gefitinib plus chemotherapy versus placebo plus chemotherapy in EGFR-mutation-positive non-small-cell lung cancer after progression on first-line gefitinib (IMPRESS): a phase 3 randomised trial. Lancet Oncol. 16, 990-998.

Soria, J.C., et al., 2016. Gefitinib/chemotherapy vs chemotherapy in EGFR mutation-positive NSCLC after progression on 1st line gefitinib (IMPRESS study): Final overall survivall analysis. Ann. Oncol. 27 (6), 416-454, http://dx doi.org/10.1093/annonc/mdw383.

Spigel, D.R., et al., 2016. Total mutation burden (TMB) in lung cancer (LC) and relationship with response to PD-1/PD-L1 targeted therapies. J. Clin. Oncol. 34, suppl; abstr 9017

Stahel, R.A., et al., 2015. A phase II trial of erlotinib (E) and bevacizumab (B) in patients with advanced non-small-cell lung cancer (NSCLC) with activating epidermal growth factor receptor (EGFR) mutations with and without T790 M mutation. In: Presentedat 2015 European Cancer Congress; Sep 25-29, Vienna, Abstract 3BA.

Sugawara, S., Oizumi, S., Minato, K., et al., 2015. Randomized phase II study of concurrent versus sequential alternating gefitinib and chemotherapy in previously untreated non-small cell lung cancer with sensitive EGFR mutations: NEJ005/TCOG0902. Ann. Oncol, 26, 888-894.

Takahama, T., Sakai, K., Takeda, M., et al., 2016. Detection of the T790M mutation of EGFR in plasma of advanced non-small cell lung cancer patients with acquired resistance to tyrosine kinase inhibitors (West Japan oncology group 8014LTR study). Oncotarget 7, 58492-58499. 
Tan, D.S.-W., et al., 2016. Updated results of a phase 1 study of EGF816, a third-generation, mutant-selective EGFR tyrosine kinase inhibitor (TKI), in advanced non-small cell lung cancer (NSCLC) harboring T790M. J. Clin. Oncol. 34, suppl; abstr 9044.

Thompson, J.C., Yee, S.S., Troxel, A.B., et al., 2016. Detection of therapeutically targetable driver and resistance mutations in lung cancer patients by next-generation sequencing of cell-free circulating tumor DNA. Clin. Cancer Res. 22, 5772-5782.

Thress, K.S., Brant, R., Carr, T.H., et al., 2015. EGFR mutation detection in ctDNA from NSCLC patient plasma: a cross-platform comparison of leading technologies to support the clinical development of AZD9291. Lung Cancer 90, 509-515.

Togashi, Y., Masago, K., Masuda, S., et al., 2012. Cerebrospinal fluid concentration of gefitinib and erlotinib in patients with non-small cell lung cancer. Cancer Chemother. Pharmacol. 70, 399-405.

Umemura, S., Tsubouchi, K., Yoshioka, H., et al., 2012. Clinical outcome in patients with leptomeningeal metastasis from non-small cell lung cancer: Okayama Lung Cancer Study Group. Lung Cancer 77, 134-139.

Villaflor, V., Won, B., Nagy, R., et al., 2016. Biopsy-free circulating tumor DNA assay identifies actionable mutations in lung cancer. Oncotarget 7 (41), 66880-66891.

Wu, Y.L., Lee, J.S., Thongprasert, S., et al., 2013. Intercalated combination of chemotherapy and erlotinib for patients with advanced stage non-small-cell lung cancer (FASTACT-2): a randomised, double-blind trial. Lancet Oncol. 14, 777-786.

Wu, Y.L., Zhou, C., Hu, C.P., et al., 2014. Afatinib versus cisplatin plus gemcitabine for first-line treatment of Asian patients with advanced non-small-cell lung cancer harbouring EGFR mutations (LUX-Lung 6): an open-label, randomised phase 3 trial. Lancet Oncol. 15, 213-222.

Wu, Y., Liu, H., Shi, X., Song, Y., 2015. Can EGFR mutations in plasma or serum be predictive markers of non-small-cell lung cancer? A meta-analysis. Lung Cancer 88, 246-253.

Wu, Y.L., et al., 2017. BRAIN: a phase III trial comparing WBI and chemotherapy with icotinib in NSCLC with brain metastases harboring EGFR mutations (CTONG 1201). J. Thorac. Oncol. 12 (1), S6

Yang, J.C., Wu, Y.L., Schuler, M., et al., 2015a. Afatinib versus cisplatin-based chemotherapy for EGFR mutation-positive lung adenocarcinoma (LUX-Lung 3 and LUX-Lung 6): analysis of overall survival data from two randomised, phase 3 trials. Lancet Oncol. 16, 141-151.
Yang, J., Zhou, Q., Yan, H.H., et al., 2015b. A randomized controlled trial of erlotinib versus gefitinib in advanced non-small-cell lung cancer harboring EGFR mutations (CTONG0901). 16th World Conference on Lung Cancer; Denver, CO, USA: Sept 6-9, Abstr 16.13

Yang, J.C., Sequist, L.V., Geater, S.L., et al., 2015c. Clinical activity of afatinib in patients with advanced non-small-cell lung cancer harbouring uncommon EGFR mutations: a combined post-hoc analysis of LUX-Lung 2, LUX-Lung 3 , and LUX-Lung 6. Lancet Oncol. 16, 830-838.

Yang, J.C., Sequist, L.V., Zhou, C., et al., 2016a. Effect of dose adjustment on the safety and efficacy of afatinib for EGFR mutation-positive lung adenocarcinoma: post hoc analyses of the randomized LUX-Lung 3 and 6 trials. Ann. Oncol. 27, 2103-2110.

Yang, J.C.H., et al., 2016b. Osimertinib activity in patients with leptomeningeal (LM) disease from non-small cell lung cancer (NSCLC): updated results from BLOOM, a phase I study. Int. J. Clin. Oncol. 34, suppl; abstr 9002.

Yoon, H.J., Lee, H.Y., Lee, K.S., et al., 2012. Repeat biopsy for mutational analysis of non-small cell lung cancers resistant to previous chemotherapy: adequacy and complications. Radiology 265, 939-948.

Yu, H.A., Arcila, M.E., Rekhtman, N., et al., 2013. Analysis of tumor specimens at the time of acquired resistance to EGFR-TKI therapy in 155 patients with EGFR-mutant lung cancers. Clin. Cancer Res. 19, 2240-2247.

Yu, H.A., et al., 2016. Antitumor activity of ASP8273 $300 \mathrm{mg}$ in subjects with EGFR mutation-positive non-small cell lung cancer: interim results from an ongoing phase 1 study. Int. J. Clin. Oncol. 34, suppl; abstr 9050.

Zhang, Q., Zhang, X., Yan, H., et al., 2016a. Effects of epidermal growth factor receptor-tyrosine kinase inhibitors alone on EGFR-mutant non-small cell lung cancer with brain metastasis. Thorac. Cancer 7, 648-654.

Zhang, L., Zhao, H., Hu, B., et al., 2016b. First-in-human study of A, a novel irreversible, mutant-selective EGFR inhibitor in patients with 1 st generation EGFR TKI-resistant non-small cell lung cancer (NSCLC). Ann. Oncol. 27 (Suppl 6), 3590.

Zhao, J., Chen, M., Zhong, W., et al., 2013. Cerebrospinal fluid concentrations of gefitinib in patients with lung adenocarcinoma. Clin. Lung Cancer 14, 188-193.

Zhou, C., Wu, Y.L., Chen, G., et al., 2011. Erlotinib versus chemotherapy as first-line treatment for patients with advanced EGFR mutation-positive non-small-cell lung cancer (OPTIMAL, CTONG-0802): a multicentre, open-label, randomised, phase 3 study. Lancet Oncol. 12, 735-742. 